\title{
Estimation for a bivariate survival model based on exponential distributions with a location parameter ${ }^{\dagger}$
}

\author{
Yeon Woong Hong ${ }^{1}$ \\ ${ }^{1}$ School of Management and Tourism, Dongyang University \\ Received 14 May 2014, revised 17 June 2014, accepted 1 July 2014
}

\begin{abstract}
A bivariate exponential distribution with a location parameter is proposed as a model for a two-component shared load system with a guarantee time. Some statistical properties of the proposed model are investigated. The maximum likelihood estimators and uniformly minimum variance unbiased estimators of the parameters, mean time to failure, and the reliability function of system are obtained with unknown guarantee time. Simulation studies are given to illustrate the results.
\end{abstract}

Keywords: Bivariate exponential distribution, reliability, uniformly minimum variance unbiased estimator.

\section{Introduction}

The exponential distribution has been widely used in life testing and reliability. To improve the reliability of a system, it is a common practice to connect several components in parallel. If component failures are mutually statistically independent, the estimation of the system reliability is straightforward. In some situations, however, all the components share the load during the mission time and the failure rate of the surviving components may increase due to increased load when a component fails. Studies in software reliability and fault-tolerant software have found that the reliability of software is a function of the system load as well as the processing time (see Iyer and Rossetti, 1985). In fault-tolerant design where multiple processors share the incoming load, the failure of one processor does increase the workload, and often the failure rate of the surviving processor (see Musa et al., 1987). Statistically dependent failures are typical for such systems. To correctly estimate the reliability of such systems, the increase of failure rate of the surviving components has to be considered.

Freund (1961) proposed a bivariate extension of the exponential distribution by allowing the failure rate of the surviving component to be changed after the failure of another component. Freund model can be viewed as a simple load-share model for a two-component system. He was one of the first who introduced a physically motivated model.

Weier (1981) obtained Bayes estimators for the parameters and reliability for the Freund model using a reparametrization. Lin et al. (1993) extended the Freund's bivariate model

$\dagger$ This research was supported by Dongyang university research fund.

${ }^{1}$ Professor, School of Management and Tourism, Dongyang University, Kyungbook 720-711, Korea. E-mail: hong@dyu.ac.kr 
to a the trivarite and multivariate models for nonrepairable systems. Kim and Kvam (2004) extended the Freund's model to the general k component case and introduced load-share parameters using a reparameterization as in Weier (1981). They found maximum likelihood estimators of the parameters and derived a likelihood ratio test for testing equality of parameters against the alternative that they are monotone. Singh et al. (2008) obtained the maximum likelihood and Bayes estimates of the parameters of a k-components load-sharing parallel system in which some of the components follow an exponential distribution.

Scheuer (1988) obtained the reliability of m-out-of-n system under the assumption that failure of a component changes the failure rates of the surviving components. Shao and Lamberson (1991) considered a Markov model for analyzing the reliability and availability of an n-unit shared-load repairable k-out-of-n system with imperfect switching. Pham (1992) developed the reliability function of two-component shared-load system with imperfect coverage. Liu (1998) presented a generalized model to calculate the reliability of a load-sharing k-out-of-n system for arbitrary distributions. Cha (2009) obtained the maximum likelihood estimates and the modified maximum likelihood estimates of mean time to failure and reliability function for shared load model by using censored system life data. Cho (2012) obtained maximum likelihood estimates for parameters of Freund's model under the system level life testing.

However, in most research works for exponential shared-load model, they have assumed that location parameters are zero. Clearly, this is not always the case (see Lawless, 1982). For this reason in the present paper we suppose that location parameters are non-zero. In this paper, we propose a generalized Freund's model having a location parameter for a two-component shared-load system with a guarantee time and consider statistical inferences on the parameters, system mean time to failure (MTTF) and reliability. We obtain the maximum likelihood estimators (MLE's) and the uniformly minimum variance unbiased estimators (UMVUE's) of the parameters, MTTF, and the reliability.

\section{Bivariate exponential distribution and its properties}

Let $X$ and $Y$ represent the lifetimes of components $C_{1}$ and $C_{2}$ of a two-component parallel system. Suppose $X \sim E\left(\alpha^{-1}, \mu\right)$ and $Y \sim E\left(\beta^{-1}, \mu\right)$, where $E\left(\alpha^{-1}, \mu\right)$ denotes the two-parameter exponential distribution with probability density function(pdf) $g(x)=$ $\alpha \exp \{-\alpha(x-\mu)\}, x>\mu$. Further, assume that the failure rate of component $C_{2}\left(C_{1}\right)$ changes to $\beta^{\prime}\left(\alpha^{\prime}\right)$ from $\beta(\alpha)$ if $C_{1}\left(C_{2}\right)$ fails first, and the location parameter $\mu$ does not change. Applying the approach of Freund (1961), we obtain the joint density of $(X, Y)$

$$
f(x, y)=\left\{\begin{array}{l}
\alpha \beta^{\prime} \cdot \exp -\beta^{\prime}(y-\mu)-\left(\alpha+\beta-\beta^{\prime}\right)(x-\mu), y>x>\mu \\
\alpha^{\prime} \beta \cdot \exp -\alpha^{\prime}(x-\mu)-\left(\alpha+\beta-\alpha^{\prime}\right)(y-\mu), x>y>\mu
\end{array}\right.
$$

which is a bivariate extension of the two-parameter exponential distribution and does not allow the simultaneous failures. For convenience, we denote $(X, Y) \sim B V E\left(\alpha, \beta, \alpha^{\prime}, \beta^{\prime}, \mu\right)$ if a random vector $(X, Y)$ is distributed as a BVE with the joint density function (2.1). Of course when $\mu=0,(2.1)$ coincides with Freund model. Freund has given some basic properties for $B V E\left(\alpha, \beta, \alpha^{\prime}, \beta^{\prime}, 0\right)$. Modifying his derivations, the moment generating function of $B V E$ 
$\left(\alpha, \beta, \alpha^{\prime}, \beta^{\prime}, \mu\right)$ is given by

$$
\begin{aligned}
m(s, t) & =\frac{\alpha+\beta}{1-(s+t) /(\alpha+\beta)}\left(\frac{\beta}{1-s / \alpha^{\prime}}+\frac{\alpha}{1-t / \beta^{\prime}}\right) \exp \{\mu(s+t)\} \\
& =(\alpha+\beta)\left\{1+\frac{s+t}{\alpha+\beta}+\left(\frac{s+t}{\alpha+\beta}\right)^{2}+\cdots\right\} \\
& \times\left\{\beta\left[1+\frac{s}{\alpha^{\prime}}+\left(\frac{s}{\alpha^{\prime}}\right)^{2}+\cdots\right]+\alpha\left[1+\frac{t}{\beta^{\prime}}+\left(\frac{t}{\beta^{\prime}}\right)^{2}+\cdots\right]\right\} \\
& \times\left\{1+\mu s+\frac{(\mu s)^{2}}{2 !}+\cdots\right\}\left\{1+\mu t+\frac{(\mu t)^{2}}{2 !}+\cdots\right\} .
\end{aligned}
$$

Selecting the appropriate coefficients of this power series in $s$ and $t$, it follows that

$$
\begin{gathered}
E[X]=\frac{\alpha^{\prime}+\beta}{\alpha^{\prime}(\alpha+\beta)}+\mu, E[Y]=\frac{\alpha+\beta^{\prime}}{\beta^{\prime}(\alpha+\beta)}+\mu, \\
\operatorname{Var}[X]=\frac{\alpha^{\prime 2}+2 \alpha \beta+\beta^{2}}{\alpha^{\prime 2}(\alpha+\beta)^{2}}, \operatorname{Var}[Y]=\frac{\alpha^{2}+2 \alpha \beta+\beta^{\prime 2}}{\beta^{\prime 2}(\alpha+\beta)^{2}}, \operatorname{Cov}(X, Y)=\frac{\alpha^{\prime} \beta^{\prime}-\alpha \beta}{\alpha^{\prime} \beta^{\prime}(\alpha+\beta)^{2}} .
\end{gathered}
$$

Integrating (2.1), in turn, with respect to $y$ and $x$, we obtain that

$$
\begin{array}{r}
f_{X}(x)=\frac{\left(\alpha-\alpha^{\prime}\right)(\alpha+\beta)}{\alpha+\beta-\alpha^{\prime}} \exp \left\{-(\alpha+\beta)(x-\mu)+\frac{\alpha^{\prime} \beta}{\alpha+\beta-\alpha^{\prime}} \exp \left\{-\alpha^{\prime}(x-\mu)\right\}\right\}, \\
x>\mu, \alpha+\beta \neq \alpha^{\prime}, \\
f_{Y}(y)=\frac{\left(\beta-\beta^{\prime}\right)(\alpha+\beta)}{\alpha+\beta-\beta^{\prime}} \exp \left\{-(\alpha+\beta)(y-\mu)+\frac{\alpha^{\prime} \beta}{\alpha+\beta-\beta^{\prime}} \exp \left\{-\beta^{\prime}(y-\mu)\right\}\right\}, \\
y>\mu, \alpha+\beta \neq \beta^{\prime},
\end{array}
$$

which are mixtures of two 2-parameter exponential distributions.

If $\alpha+\beta=\alpha^{\prime}$, then

$$
f_{X}(x)=\{\alpha+\beta(\alpha+\beta)(x-\mu)\} \exp \{-(\alpha+\beta)(x-\mu)\}, x>\mu
$$

and if $\alpha+\beta=\beta^{\prime}$

$$
f_{Y}(x)=\{\beta+\alpha(\alpha+\beta)(y-\mu)\} \exp \{-(\alpha+\beta)(y-\mu)\}, y>\mu,
$$

which are mixtures of exponential and gamma distributions with the common location parameter $\mu$. Generally, these marginal distributions are not two-parameter exponential. However, in the special cases where $\alpha=\alpha^{\prime}$ and $\beta=\beta^{\prime}, X$ and $Y$ are independently distributed as $E(1 / \alpha, \mu)$ and $E(1 / \beta, \mu)$, respectively. When two components are installed in parallel, 
the system reliability at mission time $t$ is

$$
\begin{aligned}
R(t)= & P[\max (X, Y)>t] \\
= & \int_{\mu}^{t} P\left(T_{2}>t \mid T_{1}=t\right) f_{X}\left(t_{1}\right) d t_{1}+\int_{\mu}^{t} P\left(T_{1}>t \mid T_{2}=t\right) f_{Y}\left(t_{2}\right) d t_{2}+P\left(T_{1}>t, T_{2}>t\right) \\
= & \frac{\alpha}{\alpha+\beta-\beta^{\prime}} \exp \left\{-\beta^{\prime}(t-\mu)\right\}+\frac{\beta}{\alpha+\beta-\alpha^{\prime}} \exp \left\{-\alpha^{\prime}(t-\mu)\right\} \\
& +\left(1-\frac{\alpha}{\alpha+\beta-\beta^{\prime}}-\frac{\beta}{\alpha+\beta-\alpha^{\prime}}\right) \exp \{-(\alpha+\beta)(t-\mu)\},
\end{aligned}
$$

for $\alpha+\beta \neq \alpha^{\prime}$ and $\alpha+\beta \neq \beta^{\prime}$. In the case of $\alpha+\beta=\alpha^{\prime}$ and $\alpha+\beta \neq \beta^{\prime}$ one obtains from $(2.2)$

$$
R(t)=\frac{\alpha}{\alpha+\beta-\beta^{\prime}} \exp \left\{-\beta^{\prime}(t-\mu)\right\}+\left(\frac{\beta-\beta^{\prime}}{\alpha+\beta-\beta}+\beta(t-\mu)\right) \exp \{-(\alpha+\beta)(t-\mu)\} .
$$

If $\alpha+\beta \neq \alpha^{\prime}$ and $\alpha+\beta=\beta^{\prime}$, then

$$
R(t)=\frac{\beta}{\alpha+\beta-\alpha^{\prime}} \exp \left\{-\alpha^{\prime}(t-\mu)\right\}+\left(\frac{\alpha-\alpha^{\prime}}{\alpha+\beta-\beta^{\prime}}+\alpha(t-\mu)\right) \exp \{-(\alpha+\beta)(t-\mu)\} .
$$

If $\alpha+\beta=\alpha^{\prime}=\beta^{\prime}$, then

$$
R(t)=\{1+(\alpha+\beta)(t-\mu)\} \exp \{-(\alpha+\beta)(t-\mu)\} .
$$

When $\mu=0,(2.2),(2.3),(2.4)$ and (2.5) coincide with (2-11), (2-12), (2-13), and (214) of Lin et al. (1993), respectively. (Note that the parametric conditions in Lin et al. (1993) include typographical errors. For example, in Equation (2-12), " $\alpha+\triangle \beta_{01}=0$ and $\triangle \alpha_{01}+\beta \neq 0 "$ should be corrected to " $\alpha+\triangle \beta_{01} \neq 0$ and $\triangle \alpha_{01}+\beta=0 "$. In Equation (2-13), " $\beta+\triangle \alpha_{01}=0$ and $\triangle \beta_{01}+\alpha \neq 0 "$ should be corrected to " $\beta+\triangle \alpha_{01} \neq 0$ and $\left.\triangle \beta_{01}+\alpha=0 "\right)$.

And the mean time to failure of the system, denoted by $M$, is given by

$$
\begin{aligned}
M & =\int_{0}^{\infty} R(t) d t=\int_{0}^{\mu} 1 d t+\int_{\mu}^{\infty} R(t) d t \\
& =\frac{1}{\alpha+\beta}\left(1+\frac{\alpha}{\beta^{\prime}}+\frac{\beta}{\alpha^{\prime}}\right)+\mu .
\end{aligned}
$$

\section{Estimation of parameters and reliability}

In this section, we consider the model (2.1) where $\alpha=\beta=1 / \theta$ and $\alpha^{\prime}=\beta^{\prime}=1 / \theta^{\prime}$. Then from (2.1), we obtain the joint density of $(X, Y)$

$$
f(x, y)=\frac{1}{\theta \theta^{\prime}} \cdot \exp \left[-\frac{2}{\theta}\{\min (x, y)-\mu\}-\frac{1}{\theta^{\prime}}\{\max (x, y)-\min (x, y)\}\right],
$$


where $\min (x, y)>\mu, \theta>0, \theta^{\prime}>0$. And from (2.2) and (2.5)

$$
R(t)=\left\{\begin{array}{l}
\frac{2 \theta^{\prime}}{2 \theta^{\prime}-\theta} \exp \left\{-\frac{t-\mu}{\theta^{\prime}}\right\}-\frac{\theta}{2 \theta^{\prime}-\theta} \exp \left\{-\frac{2(t-\mu)}{\theta}\right\}, \text { if } \theta \neq 2 \theta^{\prime}, \\
\left\{1+\frac{2(t-\mu)}{\theta}\right\} \exp \left\{-\frac{2(t-\mu)}{\theta}\right\}, \text { if } \theta=2 \theta^{\prime},
\end{array}\right.
$$

and from (2.6), we obtain the MTTF

$$
M=\theta / 2+\theta^{\prime}+\mu .
$$

If $\left(X_{i}, Y_{i}\right), i=1,2, \cdots, n$, is a random sample of size $n$ from (3.1), then the likelihood function based on this is given by

$$
L\left(\mu, \theta, \theta^{\prime}\right)=\prod_{i=1}^{n} f\left(x_{i}, y_{i}\right)=\left(\theta \theta^{\prime}\right)^{-n} \exp \left\{-\frac{2\left(t_{1}-n \mu\right)}{\theta}-\frac{t_{2}}{\theta^{\prime}}\right\}, z_{i}>\mu,
$$

where $t_{1}=\sum_{i=1}^{n} z_{i}, t_{2}=\sum_{i=1}^{n} w_{i}$, and where $z_{i}=\min \left(x_{i}, y_{i}\right), w_{i}=\max \left(x_{i}, y_{i}\right)-\min \left(x_{i}, y_{i}\right)$. (A lower case letter will denote the realized value of a random variable denoted by the corresponding capital letter.) In the case of $\theta \neq 2 \theta^{\prime}$, the MLE's of $\mu, \theta$ and $\theta^{\prime}$ are given, respectively, by

$$
\widehat{\mu}=Z_{(1)}, \widehat{\theta}=\left(2 T_{1}-2 n Z_{(1)}\right) / n, \widehat{\theta}^{\prime}=T_{2} / n
$$

where $Z_{(1)}=\min \left(Z_{1}, Z_{2}, \cdots, Z_{n}\right)$. In the case of $\theta=2 \theta^{\prime}$, letting $\theta^{\prime}=\theta / 2$ in (3.4), we obtain the MLE's of $\mu$ and $\theta$ as

$$
\widehat{\mu}=Z_{(1)}, \widehat{\theta}=\left(T_{1}+T_{2}-n Z_{(1)}\right) / n .
$$

Employing the invariance property of MLE and the expressions (3.2) and (3.3), the MLE's $\widehat{R}(t)$ and $\widehat{M}$ of $R(t)$ and MTTF can be obtained.

We now find the UMVUE's of parameters, MTTF and reliability function. Suppose that $\theta \neq 2 \theta^{\prime}$. From (3.4), it is readily seen that $\left(Z_{(1)}, T_{1}, T_{2}\right)$ is a complete sufficient for $\left(\mu, \theta, \theta^{\prime}\right)$. And from (3.1), it is well known (Lawless (1982)) that $Z_{i}$ and $W_{i}$ are independently distributed as $E(\theta / 2, \mu)$ and $E\left(\theta^{\prime}, 0\right)$, respectively. Then it is easily seen that

$$
\frac{2 n\left(Z_{(1)-} \mu\right)}{\theta / 2} \sim \chi^{2}(2), \frac{2\left(T_{1}-n Z_{(1)}\right)}{\theta / 2} \sim \chi^{2}(2 n-2), \text { and } \frac{2 T_{2}}{\theta^{\prime}} \sim \chi^{2}(2 n) .
$$

In the case of $\theta=2 \theta^{\prime}$ it is easily seen that $\left(Z_{(1)}, T_{1}+T_{2}\right)$ is a complete sufficient for $(\mu, \theta)$, and

$$
\frac{2\left(T_{1}+T_{2}-n Z_{(1)}\right)}{\theta / 2} \sim \chi^{2}(4 n-2) .
$$

Therefore, from (3.7) and (3.8), we have the following results.

Theorem 3.1 (UMVUE's of parameters and MTTF when $\mu>0$ )

1) If $\theta \neq 2 \theta^{\prime}$, then the UMVUE's of $\mu, \theta$ and $\theta^{\prime}$ are

$$
\widetilde{\mu}=Z_{(1)}-\left(T_{1}-n Z_{(1)}\right) / n(n-1), \widetilde{\theta}=2\left(T_{1}-n Z_{(1)}\right) /(n-1), \widetilde{\theta}^{\prime}=T_{2} / n .
$$


2) If $\theta=2 \theta^{\prime}$, then the UMVUE's of $\mu$ and $\theta$ are

$$
\widetilde{\mu}=Z_{1}-\left(T_{1}+T_{2}-n Z_{(1)}\right) / n(2 n-1), \widetilde{\theta}=2\left(T_{1}+T_{2}-n Z_{(1)}\right) /(2 n-1) .
$$

3) The UMVUE of MTTF is

$$
\widetilde{M}=\left(T_{1}+T_{2}\right) / n
$$

Theorem 3.2 Let $U=\min \left(T_{1}, T_{2}\right), V=\max \left(T_{1}, T_{2}\right)$, and $S=T_{1}+T_{2}$. Then, for $n>2$, the UMVUE of $R(t)$ is given by

1) Case of $\theta \neq 2 \theta^{\prime}$;

$$
\begin{aligned}
& \widetilde{R}(t)=\frac{\left(t_{2}+z_{(1)}-t\right)^{n-1}}{t_{2}^{n-1}}+\frac{A_{1}}{n-2} \int_{0}^{t-z_{(1)}}\left(t_{2}-s\right)^{n-2}\left(t_{1}+z_{(1)}-t+s\right)^{n-2} d s, \\
& \text { if } z_{(1)}<t<\min \left(t_{1}, t_{2}\right)+z_{(1)}, \\
& \widetilde{R}(t)=\frac{A_{1}}{n-2} \int_{0}^{t_{2}}\left(t_{2}-s\right)^{n-2}\left(t_{1}+z_{(1)}-t+s\right)^{n-2} d s \text {, if } t_{2}+z_{(1)}<t \leq t_{1}+z_{(1)}, \\
& \widetilde{R}(t)=\frac{\left(t_{2}+z_{(1)}-t\right)^{n-1}}{n t_{2}^{n-1}}+\frac{A_{1}}{n-1} \int_{0}^{t_{1}}\left(t_{1}-s\right)^{n-3}\left(t_{2}+z_{(1)}-t+s\right)^{n-1} d s, \\
& \text { if } t_{1}+z_{(1)}<t \leq t_{2}+z_{(1)} \text {, } \\
& \widetilde{R}(t)=\frac{A_{1} B(n, n-2)}{n-1}\left(t_{1}+t_{2}+z_{(1)}-t\right)^{2 n-3} \text {, if } \max \left(t_{1}, t_{2}\right)+z_{(1)}<t \leq t_{1}+t_{2}+z_{1} \text {, }
\end{aligned}
$$

2) Case of $\theta \neq 2 \theta^{\prime}$;

$$
\begin{aligned}
\widetilde{R}(t) & =1, \text { if } t<z_{(1)} \\
& =\frac{2(n-1)^{2}}{n}\left(\frac{t-z_{(1)}}{s}\right)\left(1-\frac{t-z_{(1)}}{s}\right)^{2 n-3}+\frac{n-1}{n}\left(1-\frac{t-z_{(1)}}{s}\right)^{2 n-2}, \text { if } z_{(1)} \leq t \leq z_{(1)}+s \\
& =0, \text { otherwise. }
\end{aligned}
$$

Proof of 1: Let $\xi_{1}$ be the one of the observations in $Z_{i}, i=1,2, \cdots, n$, and $W_{1}=$ $\max \left(X_{1}, Y_{1}\right)-\min \left(X_{1}, Y_{1}\right)$. Then $I\left(W_{1}+\xi_{1}>t\right)$ is an unbiased estimator of $R(t)$, where $I(\cdot)$ denotes the usual indicator function. Since $\left(T_{1}, T_{2}, Z_{(1)}\right)$ is complete sufficient statistic, the conditional expectation $E\left[I\left(W_{1}+\xi_{1}>t\right) \mid T_{1}=t_{1}, T_{2}=t_{2}, Z_{(1)}=z_{(1)}\right]$ is the UMVUE. By noting that $\left(\xi_{1}, T_{1}, Z_{(1)}\right)$ and $\left(W_{1}, T_{2}\right)$ are independent, the conditional joint density $f\left(w_{1}, \xi_{1} \mid t_{1}, t_{2}, z_{(1)}\right)$ of $\left(w_{1}, \xi_{1}\right)$ given $\left(T_{1}, T_{2}, Z_{(1)}\right)$ is the product of two conditional distributions $g\left(\xi_{1} \mid t_{1}, z_{(1)}\right)$ and $h\left(w_{1} \mid t_{2}\right)$. Applying the approach of Laurent (1963), we obtain the conditional density of $\xi_{1}$ given $\left(T_{1}, Z_{(1)}\right)$ as

$$
g\left(\xi_{1} \mid t_{1}, z_{(1)}\right)=\left\{\begin{array}{l}
1 / n, \xi_{1}=z_{(1)}, \\
(n-1)(n-2)\left(t_{1}+z_{(1)}-\xi_{1}\right)^{n-3} / n t_{1}^{n-2}, z_{(1)}<\xi_{1} \leq t_{1}+z_{(1)}, \\
0, \text { otherwise. }
\end{array}\right.
$$


And the conditional density of $w_{1}$ given $T_{2}$ is easily obtained as

$$
h\left(w_{1} \mid t_{2}\right)=\left\{\begin{array}{l}
(n-1)\left(t_{2}-w_{1}\right)^{n-2} / t_{2}^{n-1}, 0<w_{1}<t_{2}, \\
0, \text { otherwise }
\end{array}\right.
$$

From (3.9) and (3.10), the conditional joint density of $\left(w_{1}, \xi_{1}\right)$ given $\left(T_{1}, T_{2}, Z_{(1)}\right)$ is

$$
\begin{aligned}
f\left(w_{1}, \xi_{1} \mid t_{1}, t_{2}, z_{(1)}\right)= & g\left(\xi_{1} \mid t_{1}, z_{(1)}\right) \cdot h\left(w_{1} \mid t_{2}\right) \\
& =\left\{\begin{array}{l}
A_{1}\left(t_{1}+z_{(1)}-\xi_{1}\right)^{n-3}\left(t_{2}-w_{1}\right)^{n-2}, \text { if } z_{(1)}<\xi_{1} \leq t_{1}+z_{(1)}, 0<w_{1}<t_{2}, \\
A_{2}\left(t_{2}-w_{1}\right)^{n-2}, \text { if } z_{(1)}=\xi_{1}, 0<w_{1}<t_{2} .
\end{array}\right.
\end{aligned}
$$

According to the Rao-Blackwell-Lehmann-Scheffe' theorem, the UMVUE of $R(t)$ is given by

$$
\widetilde{R}(t)=\iint I\left(w_{1}+\xi_{1}>t\right) f\left(w_{1}, \xi_{1} \mid t_{1}, t_{2}, z_{(1)}\right) d w_{1} d \xi_{1} .
$$

The stated results can be obtained by evaluating the integral of (3.11).

Proof of 2: We now find the UMVUE of $R(t)$ when $\theta=2 \theta^{\prime}$. Let $S=T_{1}+T_{2}-n Z_{(1)}$. Let $\xi_{1}$ be the one of the observations in $Z_{i}, i=1,2 \cdots, n$, from $E(\theta, \mu)$ and $\xi_{2}$ be the one of the observations in $W_{i}, i=1,2, \cdots, n$, from $E(\theta, 0)$. The conditional distribution of $\left(\xi_{1}, \xi_{2}\right)$ given $\left(S, Z_{(1)}\right)=\left(s, z_{(1)}\right)$ is given by (see Appendix)

$$
p\left(\xi_{1}, \xi_{2} \mid s, z_{(1)}\right)=\left\{\begin{array}{l}
1 / n, \xi_{1}=z_{(1)}, \\
A_{3}\left(s+z_{(1)}-\xi_{1}-\xi_{2}\right)^{2 n-4}, z_{(1)}<\xi_{1}<z_{(1)}+s-\xi_{2}, 0<\xi_{2}<s \\
0, \text { otherwise }
\end{array}\right.
$$

where $A_{3}=2(n-1)^{2}(2 n-3) / n s^{2 n-2}$. Thus the UMVUE $\widetilde{R}(t)=E\left[I\left(\xi_{1}+\xi_{2}>t\right) \mid S=\right.$ $\left.s, Z_{(1)}=z_{(1)}\right]$ of $R(t)$ can be obtained by evaluating the integral

$$
\widetilde{R}(t)=\iint I\left(\xi_{1}+\xi_{2}>t\right) p\left(\xi_{1}, \xi_{2} \mid s, z_{(1)}\right) d \xi_{1} d \xi_{2}
$$

\section{Simulations}

In this sub-section, we compare the relative bias and the mean square error(MSE) of the MLE and UMVUE of $R(t)$ for moderate sample sizes $\mathrm{n}=(10,20,30)$ through Monte Carlo simulation. Estimates of the relative bias and MSE were obtained from 1,000 trials with $\mu=(0,5), \theta=10$, and $\theta^{\prime}=(2.5,5.0,7.5)$. In Tables 1 and 2 , the relative bias(in percentage) of MLE(or UMVUE) is calculated from $100 \times(\widehat{R}(t)$ or $\widetilde{R}(t)-R(t)) / R(t)$, and REF represents the relative efficiency of UMVUE over MLE, that is, REF $=$ MSE $(\widehat{R}(t))$ /MSE $(\widetilde{R}(t))$. Although $\widetilde{R}(t)$ is known to be unbiased, its estimated relative bias is recorded as a check on the computations.

In general, as expected, the estimated absolute relative bias of UMVUE is found to be considerably smaller than the estimated absolute relative bias of MLE. The estimated absolute relative bias and MSEs of the two estimators tend to decrease as n increases. Furthermore, the MSEs of both estimates appear to be nearly equal. 
Table 4.1 Estimates of relative bias, MSE and efficiency $(\theta=10, \mu=5, t=6.0)$

\begin{tabular}{ccccccccc}
\hline \hline \multirow{2}{*}{$\theta^{\prime}$} & \multirow{2}{*}{$R(t)$} & \multirow{2}{*}{$\mathrm{n}$} & \multicolumn{2}{c}{ relative bias of } & & \multicolumn{2}{c}{ MSE of } & \multirow{2}{*}{ REF } \\
\cline { 4 - 5 } & & & MLE & UMVUE & & MLE & UMVUE & \\
\hline \multirow{2}{*}{2.5} & \multirow{2}{*}{0.9671} & 10 & -1.623 & 0.145 & & 0.0087 & 0.0095 & 0.916 \\
& & 20 & -0.910 & -0.124 & & 0.0063 & 0.0062 & 1.016 \\
& & 30 & -0.724 & -0.083 & & 0.0041 & 0.0040 & 1.025 \\
\hline \multirow{2}{*}{5} & \multirow{2}{*}{0.9825} & 10 & -1.679 & 0.173 & & 0.0098 & 0.0096 & 1.021 \\
& & 20 & -1.008 & -0.153 & & 0.0067 & 0.0062 & 1.081 \\
& & 30 & -0.733 & -0.102 & & 0.0044 & 0.0041 & 1.073 \\
\hline \multirow{2}{*}{7.5} & \multirow{2}{*}{0.9881} & 10 & -1.700 & 0.192 & & 0.0099 & 0.0097 & 1.021 \\
& & 20 & -1.063 & -0.172 & & 0.0061 & 0.0056 & 1.089 \\
& & 30 & -0.789 & -0.111 & & 0.0048 & 0.0031 & 1.548 \\
\hline
\end{tabular}

Table 4.2 Estimates of relative bias, MSE and efficiency $(\theta=10, \mu=0, t=1.0)$

\begin{tabular}{|c|c|c|c|c|c|c|c|}
\hline \multirow{2}{*}{$\theta^{\prime}$} & \multirow{2}{*}{$R(t)$} & \multirow{2}{*}{$\mathrm{n}$} & \multicolumn{2}{|c|}{ relative bias of } & \multicolumn{2}{|c|}{ MSE of } & \multirow{2}{*}{$\mathrm{REF}$} \\
\hline & & & MLE & UMVUE & MLE & UMVUE & \\
\hline \multirow{3}{*}{2.5} & \multirow{3}{*}{0.9671} & 10 & -1.799 & 0.238 & 0.0086 & 0.0095 & 0.905 \\
\hline & & 20 & -0.827 & -0.124 & 0.0062 & 0.0051 & 1.216 \\
\hline & & 30 & -0.672 & -0.062 & 0.0023 & 0.0020 & 1.150 \\
\hline \multirow{3}{*}{5} & \multirow{3}{*}{0.9825} & 10 & -1.842 & 0.244 & 0.0098 & 0.0097 & 1.010 \\
\hline & & 20 & -0.885 & -0.122 & 0.0067 & 0.0063 & 1.063 \\
\hline & & 30 & -0.692 & -0.071 & 0.0026 & 0.0021 & 1.238 \\
\hline \multirow{3}{*}{7.5} & \multirow{3}{*}{0.9881} & 10 & -1.913 & 0.253 & 0.0098 & 0.0107 & 0.916 \\
\hline & & 20 & -0.901 & -0.142 & 0.0070 & 0.0065 & 1.077 \\
\hline & & 30 & -0.617 & -0.091 & 0.0031 & 0.0025 & 1.240 \\
\hline
\end{tabular}

\section{Conclusions}

In this paper, we proposed a generalized Freund's model having a location parameter for a two-component shared-load system with a non-zero guarantee time. We obtained the MLE's and the UMVUE's of the parameters, MTTF, and the reliability of the model.

\section{Appendix}

\section{Derivation of formula (3.12)}

Let $Z^{*}=\left(Z_{1}^{*}, Z_{2}^{*}, \cdots, Z_{n-1}^{*}\right)$ be the subsample obtained by deleting $\xi_{1}$ from $Z_{i}, i=$ $1,2, \cdots, n$, and $W^{*}=\left(W_{1}^{*}, W_{2}^{*}, \cdots, W^{*}{ }_{n-1}\right)$ be the subsample obtained by deleting $\xi_{2}$ from $W_{i}, i=1,2, \cdots, n$. Finally let

$$
Z_{(1)}^{*}=\min \left(Z_{1}^{*}, Z_{2}^{*}, \cdots, Z_{n-1}^{*}\right)
$$

and

$$
S^{*}=\sum_{i=1}^{n-1} Z_{i}^{*}+\sum_{i=1}^{n-1} W_{i}^{*}-(n-1) Z_{(1)}^{*} .
$$

Since $\frac{2 n\left(Z_{(1)}-\mu\right)}{\theta / 2} \sim \chi^{2}(2)$ and $\frac{2 s}{\theta / 2} \sim \chi^{2}(4 n-2)$, we can show that

$$
q\left(s, z_{(1)}\right)=n s^{2 n-2} \exp \left\{-\left(s+n\left(z_{(1)}-\mu\right)\right) / \theta\right\} / \theta^{2 n} \Gamma(2 n-1) .
$$


Then the joint pdf of $\left(\xi_{1}, \xi_{2}, S^{*}, Z_{(1)}^{*}\right)$ is

$$
\begin{aligned}
& p\left(\xi_{1}, \xi_{2}, s^{*}, z_{(1)}^{*}\right)=f_{z}\left(\xi_{1}\right) f_{w}\left(\xi_{2}\right) q\left(s^{*}, z_{(1)}^{*}\right) \\
& \quad=(n-1) s^{* 2 n-4} \exp \left\{-\left(\xi_{1}-\mu+\xi_{2}+s^{*}+(n-1)\left(z_{(1)}^{*}-\mu\right)\right) / \theta\right\} / \theta^{2 n} \Gamma(2 n-3),
\end{aligned}
$$

if $\xi_{1}>\mu, \xi_{2}>0, s^{*}>0, z_{(1)}^{*}>\mu$.

Consider the case where $\xi_{1}>Z_{(1)}$. In this case $Z_{(1)}^{*}=Z_{(1)}$, and $S^{*}=S-\left(\xi_{1}-Z_{(1)}\right)-\xi_{2}$. The joint pdf of $\left(\xi_{1}, \xi_{2}, S^{*}, Z_{(1)}^{*}\right)$ is, from (A.2),

$$
p\left(\xi_{1}, \xi_{2}, s^{*}, z_{(1)}^{*}\right)=\frac{(n-1)}{\theta^{2 n} \Gamma(2 n-3)}\left(s+z_{(1)}-\xi_{1}-\xi_{2}\right)^{2 n-4} \exp \left\{-\frac{s+n\left(z_{(1)}-\mu\right)}{\theta}\right\},
$$

if $\mu<z_{(1)}<\xi_{(1)}<z_{(1)}+s-\xi_{2}, 0<\xi_{2}<s, s>0$. From (A.1) and (A.3), the conditional distribution of $\left(\xi_{1}, \xi_{2}\right)$ given $\left(S, Z_{(1)}\right)=\left(s, z_{(1)}\right)$ is then given by formula (3.12).

\section{References}

Cha, Y. J. (2009). Reliability estimation for shared load model with guarantee time under censoring scheme. Journal of the Korean Data 83 Information Science Society, 20, 467-474.

Cho, K. (2012). Estimation of Freund model under censored data. Journal of the Korean Data 85 Information Science Society, 23, 403-409.

Freund, J. E. (1961). A bivariate extension of the exponential distribution. Journal of the American Statistical Association, 56, 971-977.

Iyer, R. K. and Rossetti, D. J. (1985). Effect of system workload on operating system reliability: A study of IBM 3081. IEEE Transactions on Software Engineering, 11, 1438-1448.

Kim H. and Kvam, P. H. (2004). Reliability estimation based on system data with an unknown load share rule. Lifetime Data Analysis, 10, 83-94.

Laurent, A. G. (1963). Conditional distribution of order statistics and distribution of the reduced $i$ th order statistic of the exponential model. Annals of Mathematical Statistics, 34, 652-657.

Lawless, J. F. (1982). Statistical models and methods for lifetime data, John Wiley \& Sons, New York.

Lin, H. H., Chen, K. H. and Wang, R. T. (1993). A multivariate exponential shared-load model. IEEE Transactions on Reliability, 42, 165-171.

Liu, H. (1998). Reliability of a load-sharing k-out-of-n:G system: Non-iid components with arbitrary distributions. IEEE Transactions on Reliability, 47, 279-284.

Musa, J. D., Iannino, A. and Okumoto, K. (1987). Software reliability: Measurement, prediction application, McGraw-Hill, Inc., New York.

Pham, H. (1992). Reliability analysis of a high voltage system with dependent failures and imperfect coverage. Reliability Engineering and System Safety, 37, 25-28.

Scheuer, E. M. (1988). Reliability of an m-out-of-n system when component failure induces higher failure rates in survivors. IEEE Transactions on Reliability, 37, 73-74.

Shao, J. and Lamberson, L. R. (1991). Modeling a shared-load k-out-of-n:G system. IEEE Transactions on Reliability. 40, 205-209.

Singh, B., Sharma, K. K. and Kumar, A. (2008). A classical and Bayesian estimation of a k-components load-sharing parallel system. Computational Statistics \& Data Analysis, 52, 5175-5185.

Weier, D. R. (1981). Bayes estimation for a bivariate survival model based on exponential distributions. Communications in Statistics-Theory and Methods, 10, 1415-1427. 